\title{
Top-down Approach to the Investigation of the Neural Basis of Geometric-optical Illusions: Understanding the Brain as a Theoretical
} Entity

\author{
Farshad Nemati, ${ }^{1, *}$ \\ ${ }^{1}$ Canadian Centre for Behavioural Neuroscience, Department of Neuroscience, University of Lethbridge, Lethbridge, Alberta, Canada \\ "Corresponding author: Farshad Nemati, Canadian Centre for Behavioural Neuroscience, Department of Neuroscience, University of Lethbridge, Lethbridge, Alberta, Canada \\ T1K3M4. Tel: +1-647350 1148, E-mail: farshad_nemati@yahoo.ca \\ Received 2015 October 08; Accepted 2017 February 08.
}

\begin{abstract}
Geometric-optical illusions have been the subjects of research interest in a number of disciplines in science. Moreover, investigation of the patients' reactions to illusory configurations has been somewhat instrumental in the understanding of impaired neurocognitive processes underlying some of the neurological and/or psychiatric disorders. Recently, neuroscientists have made some progress in understanding the neural underpinning of the geometric-optical illusions. However, a closer collaboration between psychology and neuroscience may lead to a better understanding of not only the neural basis of the illusions but the function of the brain in general. The purpose of the present analysis is to outline a sound epistemological ground for such a relationship and to demonstrate how psychological theories may potentially play a guiding role in the context of scientific discoveries in the neuroscience of illusory phenomena. In order to do so, two concepts of the "many-one" relationship between the mental and the neural states and "context-sensitivity" will be described with regard to the possible relationships between perception and brain in the context of research on illusions. In addition, the implications of the top-down strategy for research in Psychiatry will be explained and the strategy will be discussed as a path towards the unification of scientific explanations.
\end{abstract}

Keywords: Brain, Computation, Epistemology, Illusion, Perception, Psychiatric disorder, Top-down strategy

\section{Introduction}

Geometric-optical illusions are distorted perceptual representations of some geometrical configurations (1-3). Such illusions have been the subjects of interest in disciplines such as philosophy, psychology, computer science, Neuroscience and related fields (1, 4-8). In addition, the investigation of illusions has partly turned into a tool for understanding the neuro-cognitive impairments in some neurological and/or psychiatric conditions (8-12). How close this understanding would approximate the truth partly depends on how accurate our theoretical formulation of the perceptual processes underlying illusions is and to what extent these processes correspond to the neural realizers of the illusions.

Apart from philosophical debates over reducibility of theories (13-15), scientifically, it is always important to examine how theoretical frameworks in one discipline of science may provide insights for scientific discoveries in another discipline (16). Although there is such a relationship between psychology and neuroscience (17), it has not been fully developed in some of the interesting subfields of investigation such as that of geometric-optical illusions. Recently, neuroscientists have made some progress in the investigation of the neural basis of illusions (18-20) but a closer collaboration between Psychology and Neuroscience is a step towards unification of scientific explanations (21) and lead to a better understanding of not only the neural basis of the illusions but the function of the brain in general.

Such a connection has been discussed in the context of bottom-up versus top-down approach to the investigation of a variety of perceptual and cognitive phenomena $(16,17$, 22). According to a bottom-up strategy, the overall brain function is extracted from small scale findings of neural organization. The main thesis is that understanding the organization of the brain contributes to our understanding of psychological functions (23). On the other hand, the notion that psychological theories contribute to our understanding of brain functions $(17,23)$ supports a top-down strategy of research in neuroscience. In line with the latter notion, the importance of psychological units in formulating psychological theories as well as in describing the neural underpinning of psychological phenomena has been extensively discussed by Julesz and Schumer (24) and others $(17,23)$. In addition, such a top-down strategy resonate well with Marr's (25) influential proposal that the study of vision begins with the theoretical formulation of percep-

Copyright ( ) 2017, Tehran University of Medical Sciences. This is an open-access article distributed under the terms of the Creative Commons Attribution-NonCommercial 4.0 International License (http://creativecommons.org/licenses/by-nc/4.0/) which permits copy and redistribute the material just in noncommercial usages, provided the original work is properly cited. 
tion and thus moves beyond only studying neurons. The opposite view has been advocated by others who support a bottom-up strategy in research $(22,26)$.

Although the debates have been passionately continued over the years, no agreements have been reached on a preferable strategy in a given context yet. However, in reality the adoption of a top-down strategy seems to be uncommon at least among neuroscientists who study the neural basis of the illusions. This in part may be due to the lack of a clear epistemological ground for the application of such a strategy to the investigation of geometric-optical illusions. The objective of the present analysis is to provide a sound epistemological ground for the investigation of the neural basis of the illusions via a top-down strategy of research, which will clearly reemphasize the importance of Marr's proposal. It will be demonstrated how psychological theories of illusions may outline new sets of variables for research in Neuroscience at the experimental level. Finally, the application of such a strategy will be explained with respect to the investigation of neuro-cognitive impairments in some psychiatric disorders and the implications of stronger connection between Psychology and Neuroscience will be discussed with respect to the ambitious goal of explanatory unification in science.

\section{Arguments}

\subsection{A Brief Look at the Theories of Geometric-Optical Illusions}

According to Birren (27) psychologists began studying geometric-optical illusions in nineteenth century. However, originated in some of the previous theories, a very important era for the theoretical formulation of illusions began around 1960s leading to the development of very interesting theories later during the past and present centuries. Some of such theories are formulated based on the role of 2D cues in illusory configurations, explaining some illusions and leaving others unexplained. For example, the Muller-Lyer and Titchener illusions were two well-known illusions that were never explained within the same computational framework until recently (28).

Among the most influential theories some were successful in explaining the Muller-Lyer and some in explaining the Titchener illusions but there were no theories to explain both illusions based on the same principle. For instance, according to assimilation theory the Muller-Lyer illusion occurs because the horizontal line with the outward arrowheads tends to perceptually expand towards the longer contextual stimuli and the horizontal line with the inward arrowheads tends to perceptually contract in the direction of the shorter contextual stimuli (Figure 1). However, application of such averaging process was not successful in explaining the Titchener illusion $(29,30)$.
Nevertheless, a recent theory has provided a unified explanation for both illusions. According to Nemati (28), the interaction between two distinct variables of size contrast and empty space in the background of the target stimulus can predict both the Muller-Lyer and Titchener (and some others) illusions based on the same computational processes. The theory proposes that while the size of empty space interacts with the magnitude of size contrast to determine the final magnitude of illusions, it also plays a key role in providing an orientation signal for the application of computation by the perceptual system (Figure 2A and 2B).

There are other theories that have been formulated based on other perceptual processes to explain some illusions (cf 28, 32-36) for more detailed description of the theories). The main question of the present analysis is whether perceptual principles derived from these theories can guide researchers to the discovery of a common neural mechanism for the realization of such perceptual phenomena2 (31-35). In the following sections, it will be explained how a top-down strategy of research may lead to such a success in studying illusions in human population.

\subsection{Bottom-up vs. Top-down Approach}

From empirical point of view, it is obvious today that our knowledge about visual areas of the brain can enhance our ability to predict illusory experiences (36). However, two arguments will be presented in the following sections that support the role of a top-down strategy in identifying the common neural processes inferred from perceptual principles proposed by psychological theories of illusions:

Argument 2.2.1: the concept of context-sensitivity indicates that different parts of the brain may be involved in the formation of a psychological state. For example, the C-fiber activation $(\mathrm{N})$ may be the core element of "pain" perception (M) but other parts of the brain (N1, N2 ...) may be active at the same time and perhaps contribute to the perception of the "pain" $(37,38)$. The concept of context-sensitivity is consistent with the recent supporting evidence based on MEG recordings (19). It has been demonstrated that spatio-temporal activity pattern of certain brain areas is correlated with geometric-optical illusions. Such a technology by which one can reveal the unfolding activity of the brain areas over time in fact supports the notion of context-sensitivity with regard to the realization of perceptual phenomena such as geometric-optical illusions.

Argument 2.2.2: the relationship between the mental states and their neural realizers may follow a many-one pattern of realization (39). Such patterns represent the contribution of a neural state such as " $\mathrm{N}$ " to the realization 
A
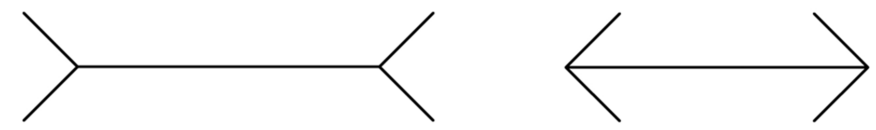

B
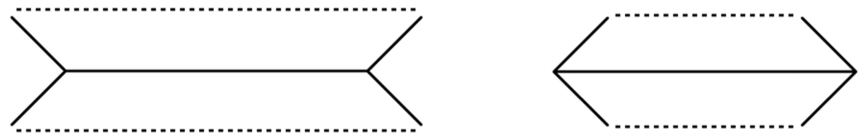

C

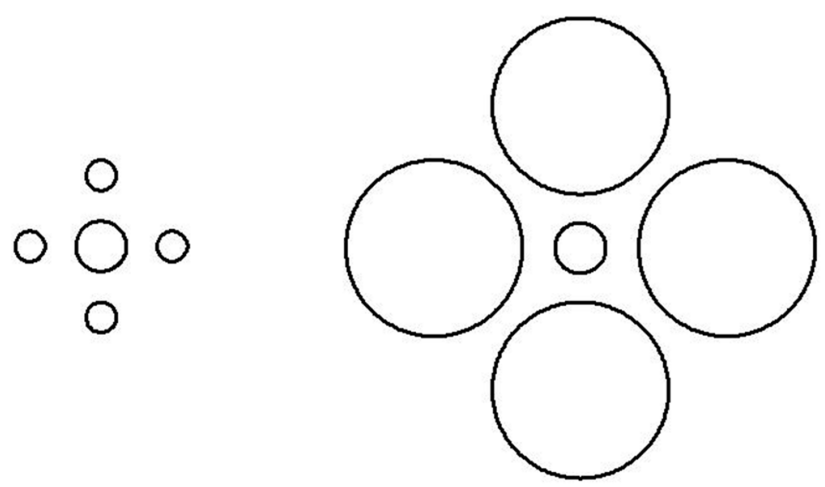

A, the Muller-Lyer configuration consists of two identical straight lines, which appear to be longer if connected to the outward arrowheads, or shorter if connected to the inward arrowheads; B, according to assimilation theory such illusion is due to the expansion or contraction of horizontal straight lines to reach the size of contextual cue represented by the dashed lines; C, However, the illusory representation of the Titchener configuration does not follow the rule described by assimilation theory. Titchener configuration consists of two identical circles in which the one surrounded by the small circles appears to be larger in size than the one surrounded by the large circles.

of a number of different mental states such as $\mathrm{m} 1, \mathrm{~m} 2, \mathrm{~m} 3$ etc. For example, "N" (that can be a functional unit such as a neuron or a neural circuit etc.) may contribute to the perception of brightness ( $\mathrm{M}$ ) and/or to that of illusory representations (M1, M2,...) in two or more drastically different geometrical configurations. The concept of many-one pattern of realization is consistent with the notion that holding a one-one relationship between a functional unit in the brain and a mental state as a general rule does not seem to be a plausible approach to the investigation of the neural basis of illusions. In fact, considering that a functional unit in the brain may contribute to the realization of various mental phenomena, it would be a very difficult task to predict which mental phenomena may be realized just by looking at the activity of a functional unit in the brain or in fact via a bottom-up strategy of research. For example, it would be very unlikely that a neuroscientist hypothesizes that a specific neural process would mediate the illusory representations of two or more configurations with quite different geometrical features (e.g. Muller-Lyer and Titchener or Muller-Lyer, Poggendorff and ZOllner config- urations) before potential common perceptual processes were demonstrated at the psychological level $(28,32)$.

Thus, a bottom-up strategy imposes some limitations on the investigation of the neural basis of various perceptual and cognitive phenomena including geometricoptical illusions (cf 24 for the application of the second argument on subjects other than geometric-optical illusions). It is important to note that diverse geometrical features of various geometric-optical configurations could deceivingly suggest the examination of different neural processes or brain areas as potential realizers of illusory representations for each configuration if a bottomup strategy were adopted. A top-down approach to the investigation of illusions does not impose such limitations as it may provide an opportunity for neuroscientists to see which neural state may satisfy the function for the realization of common perceptual processes derived from the principles of psychological (or any computational) theories of illusions. Thus, equipped with such theories, it would be much easier for a neuroscientist to discover the neural basis of perceptual processes that mediate illusions 
A

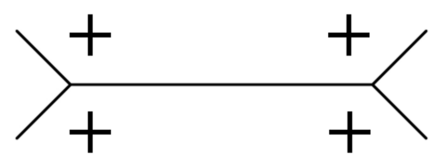

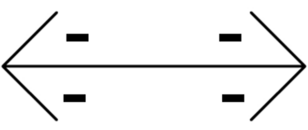

B

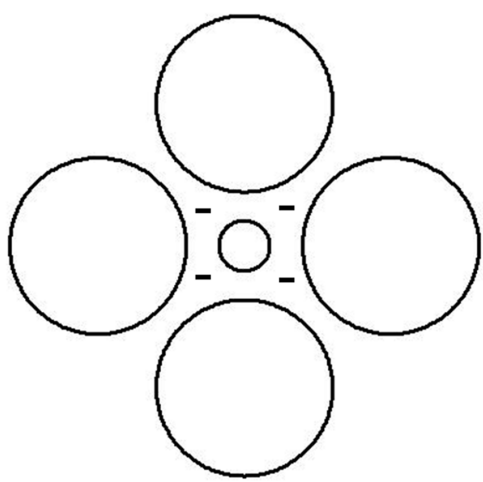

The target stimulus in the Müller-Lyer (horizontal line) and in the Titchener (central circle) configurations is overestimated when it (and/or its whole configuration) is surrounded by the larger empty space “+” and underestimated when it (and/or its whole configuration) is surrounded by the smaller empty space "-".

in such drastically different geometrical configurations. Therefore, a reasonable approach should be based on the notion that different brain areas may serve as a functional unit that contributes to the formation of some phenomena (e.g. geometric-optical illusions), while it may have other functions as well. This view clearly prescribes a topdown approach to the investigation of geometric-optical illusions. A strategy that begins with the theoretical formulation of perceptual principles from which experimental variables can be derived and their correlation with the relevant neural processes can be tested in various brain areas.

The neural realization of such perceptual processes potentially reflected in the principles of a unifying theory is conceivable based on the neurophysiological findings in the past century (40). At this level, although different geometrical features of various configurations (e.g. straight vs. curved lines etc.) may recruit distinct neural processes, the hierarchical model proposed by Hubel and Wiesel (40) provides a conceptual framework to understand the mechanism of neural integration prior to the realization of an illusory representation from such distinct configurations. According to this model, the convergence of cells in the visual areas of the brain is associated with the changes in their response profile on the way up in the hierarchy. As a result, the cells of higher order respond to more abstract features of the stimuli. Thus, the model can theoretically close the gap between the neurophysiological and perceptual analyzers proposed by Julesz and Schumers (24). In addition, the principles of a computational theory can be open to the realization of an illusory representation by the neural layers that are free from anatomical constraints required for the representation of the configuration itself (31). Therefore, the illusions can be an outcome of higher level neural activities that realize a set of computational processes. In other words, the common perceptual processes that are reflected in experimental variables (i.e. empty space/contrast, oblique intersections etc.) may mediate the formation of various illusions with distinct geometrical features (e.g. straight line in Muller-Lyer vs. curved line in Titchener illusions) as illustrated in Figure 3. Such an illusory perception of various geometric-optical configurations may be mediated by higher level neural computations in visual cortex or beyond (18).

\section{Conclusions}

Neuroscientists have begun interpreting their research findings on the illusions based on psychological 
Figure 3. A Virtual Model of Neural Underpinnings of the Illusory Configurations vs. Illusory Representations

4)

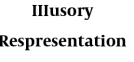

IIIusory

Respresentation

Respresentation

B

3)

2)

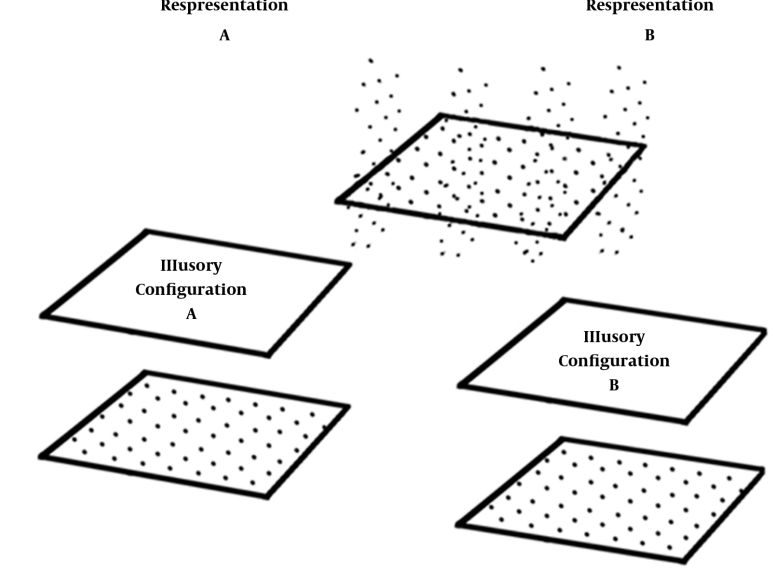

Distinct sets of neurons (dots in the layer 1) may underlie the formation of geometrical representation of two illusory configurations (A and B in layer 2) however the illusory representations of the two geometrical configurations (A and B in layer 4) may be mediated by the same set of neurons (dots in the layer 3).

principles in their recent publications (18-20). However, the correspondence between the principles that describe a perceptual phenomenon (e.g. illusory surfaces) and the functions of certain types of neurons (e.g. V4) can even guide the experimental design (41). Thus, a more direct reference to the perceptual processes inferred from such principles at the theoretical and experimental levels seems to be a reasonable move in future studies.

At the theoretical level, a top-down approach help highlighting the common perceptual processes that may correspond to some common neural processes underlying various illusions. At the experimental level, such perceptual processes can guide the researchers to define a new set of variables that might be realized by some neural processes. Such a strategy can lead to a more comprehensive understanding of the relationship between brain and illusions instead of correlating brain activities with more basic geometrical features of only one illusory configuration. This is reminiscence of Marr's (25) remark about the three levels of realization according to which he argues that only describing the behavior of so-called "grandmother cells"; does not help explaining vision unless more global features of such a behavior is theoretically formulated in terms of the task the brain is encountering. In fact, we understand the brain as an entity that serves as the realizer of specific functions such as learning, memory, or transforming the sensory inputs into perceptual representations such as geometric-optical illusions each requiring a theoretical explanation. In other words, we understand the brain as a theoretical entity. A view that is also consistent with the assumptions underlying scientific approach to artificial intelligence (42).

One clear application of such an approach is for the studies that investigate the underlying mechanisms of psychiatric disorders. For example, the investigation of geometric-optical illusions has contributed to the understanding of brain dysfunction in disorders such as schizophrenia or autism $(8-12,43,44)$. In other words, in order to understand the brain function in patient with autism, we do not benefit much from studying the reaction of this or that area of the brain to certain illusory configurations per se. The real insight comes from what we know about the theory that is likely to explain the illusory representations in conjunction with our previous knowledge about the function of the brain in patients with autism (9). Examination of a theory that defines the relationship between the brain and a phenomenon (e.g. illusions) provides an opportunity to test the picture of the brain depicted by the theory as an entity that realizes the phenomenon instead of linking the brain areas with separate subjective experiences most likely leading to not much theoretical significance.

Finally, whether or not the theories that explain various cognitive phenomena would form a coherent picture of the brain function in the end is the big question that remains to be answered as is the case in other sciences such as theoretical physics. For instance, a similar question has remained unanswered in picturing the universe 
by physicists as they look for a theory to unify the explanations of natural phenomena at the macro vs. micro level in search of a theory of everything. However, consistent with presently dominant paradigm of science, a top-down approach to the investigation of illusions seems to put the scientists in a better position for directing their research in a right scientific path towards the ambitious goal of explanatory unification (21).

\section{References}

1. Robinson JO. The psychology of visual illusion. Courier Corporation; 2013.

2. Coren S, Girgus JS, Erlichman H, Hakstian AR. An empirical taxonomy of visual illusions. Percept Psychophys. 1976;20(2):129-37. doi: 10.3758/bf03199444.

3. Bach M, Poloschek CM. Optical illusions. Adv Clin Neurosci Rehabil. 2006;6(2):20-1.

4. Fodor JA. The modularity of mind: An essay on faculty psychology. MIT press; 1983.

5. Aglioti S, DeSouza JFX, Goodale MA. Size-contrast illusions deceive the eye but not the hand. Curr Biol. 1995;5(6):679-85. doi: 10.1016/s09609822(95)00133-3.

6. Fermuller $\mathrm{C}$, Malm H. Uncertainty in visual processes predicts geometrical optical illusions. Vision Res. 2004;44(7):727-49. [PubMed: 14751556].

7. Fermüller C, Shulman D, Aloimonos Y. The Statistics of Optical Flow. Comput Vision Image Understand. 2001;82(1):1-32. doi: 10.1006/cviu.2000.0900.

8. Weckowicz TE, Witney G. The Muller-Lyer Illusion in Schizophrenic Patients. Br J Psychiatr. 1960;106(444):1002-7. doi: 10.1192/bjp.106.444.1002.

9. Happe FG. Studying weak central coherence at low levels: children with autism do not succumb to visual illusions. A research note. $J$ Child Psychol Psychiatry. 1996;37(7):873-7. [PubMed: 8923230].

10. Ropar D, Mitchell P. Are individuals with autism and Asperger's syndrome susceptible to visual illusions?. J Child Psychol Psychiatry. 1999;40(8):1283-93. [PubMed:10604406].

11. Pessoa VF, Monge-Fuentes V, Simon CY, Suganuma E, Tavares MC. The Muller-Lyer illusion as a tool for schizophrenia screening. Rev Neurosci. 2008;19(2-3):91-100. [PubMed: 18751517].

12. King JP, Christensen BK, Westwood DA. Grasping behavior in schizophrenia suggests selective impairment in the dorsal visual pathway.J Abnorm Psychol. 2008;117(4):799-811. doi: 10.1037/a0013500. [PubMed: 19025227].

13. Kuhn TS, Hawkins D. The structure of scientific revolutions. Am JPhys. 1963;31(7):554-5.

14. Feyerabend PK. Realism, Rationalism and Scientific Method: Volume 1: Philosophical Papers. Cambridge: Cambridge University Press; 1981.

15. Fodor JA. Special sciences (or: The disunity of science as a working hypothesis). Synthese. 1974;28(2):97-115. doi:10.1007/bfo0485230.

16. McCauley RN. Intertheoretic Relations and the Future of Psychology. Philosophy Sci. 1986;53(2):179-99. doi: 10.1086/289306.

17. van Eck D. Evaluating New Wave Reductionism: The Case of Vision. $\mathrm{Br}$ JPhilosophy Sci. 2006;57(1):167-96. doi:10.1093/bjps/axi153.

18. Weidner R, Fink GR. The neural mechanisms underlying the MullerLyer illusion and its interaction with visuospatial judgments. Cereb Cortex. 2007;17(4):878-84. doi: 10.1093/cercor/bhk042. [PubMed: 16707733].

19. Weidner R, Boers F, Mathiak K, Dammers J, Fink GR. The temporal dynamics of the Muller-Lyer illusion. Cereb Cortex. 2010;20(7):1586-95. doi:10.1093/cercor/bhp217. [PubMed: 19875676].
20. Qiu J, Li H, Zhang Q, Liu Q, Zhang F. The Muller-Lyer illusion seen by the brain: an event-related brain potentials study. Biol Psychol. 2008;77(2):150-8. doi:10.1016/j.biopsycho.2007.10.002. [PubMed: 17996352].

21. Causey RL. Unity of science. 109. Springer Science \& Business Media; 2012.

22. Piccinini G. Computational explanation in neuroscience. Synthese. 2006;153(3):343-53. doi: 10.1007/s11229-006-9096-y.

23. Schouten MKD, De Jong HL. Reduction, elimination, and levels: The case of the LTP-learning link. Philosoph Psychol. 1999;12(3):237-62. doi: 10.1080/095150899105747.

24. Julesz B, Schumer RA. Early visual perception. Annu Rev Psychol. 1981;32:575-627. doi: 10.1146/annurev.ps.32.020181.003043. [PubMed: 7015998].

25. Marr D. Vision. San Francisco: H. Freeman; 1982.

26. Bickle J. Psychoneural reduction: The new wave. Mit Press; 1998.

27. Birren JE. BOEING, EG: Sensation and Perception in the History of Experimental Psychology. New York, Henry Holt, 1942. Psychosomatic Med. 1944;6(1):109.

28. Nemati F. Size and direction of distortion in geometric-optical illusions: conciliation between the Muller-Lyer and Titchener configurations. Perception. 2009;38(11):1585-600. doi: 10.1068/p6450. [PubMed: 20120258].

29. Pressey AW. A theory of the Mueller-Lyer illusion. Percept Mot Skills. 1967;25(2):569-72. doi: 10.2466/pms.1967.25.2.569. [PubMed: 6080636].

30. Earlebacher A, Sekuler R. Explanation of the Muller-Lyer illusion: Confusion theory examined.J Exp Psychol. 1969;80(3p1):462.

31. Ninio J. An algorithm that generates a large number of geometric visual illusions. J Theoretical Biol. 1979;79(2):167-201. doi: 10.1016/00225193(79)90245-5.

32. Day R. On the common stimulus condition and explanation of the Müller-Lyer, Poggendorff and Zöllner illusions: The basis for a class of geometrical illusions. Aust J Psychol. 2010;62(3):115-20. doi: 10.1080/00049530903510773.

33. Pressey AW, Murray R. Further developments in the assimilation theory of geometric illusions: The adjacency ,principle. Percept Psychophys. 1976;19(6):536-44. doi:10.3758/bf03211223.

34. Woloszyn MR. Contrasting Three Popular Explanations for the Muller-Lyer Illusion. Curr Res Psychol. 2010;1(2):102-7. doi: 10.3844/crpsp.2010.102.107.

35. Nemati F. Scientific Progress in Psychology and Neuroscience of Perception: Computation, Realization, and Reduction. Can J Exp Psychol. 2012;66(4):317.

36. Schwarzkopf DS, Song C, Rees G. The surface area of human V1 predicts the subjective experience of object size. Nat Neurosci. 2011;14(1):28-30. doi: 10.1038/nn.2706. [PubMed: 21131954].

37. Shoemaker S. Some varieties of functionalism reprinted in his Identity, Cause, and Mind. New York: Cambridge University Press; 1981.

38. Wilson RA. Two views of realization. Philosophical Stud. 2001;104(1):131.

39. Endicott RP. Many-many mappings and world structure. Am Philosophical Q. 1998;35(3):267-80.

40. Hubel DH, Wiesel TN. Receptive Fields and Functional Architecture in Two Nonstriate Visual Areas (18 and 19) of the Cat. J Neurophysiol. 1965;28:229-89. [PubMed: 14283058].

41. Cox MA, Schmid MC, Peters AJ, Saunders RC, Leopold DA, Maier A. Receptive field focus of visual area V4 neurons determines responses to illusory surfaces. Proc Natl Acad Sci U S A. 2013;110(42):17095-100. doi: 10.1073/pnas.1310806110. [PubMed: 24085849].

42. Simon HA. The sciences of the artificial. MIT press; 1996.

43. Haffenden AM, Schiff KC, Goodale MA. The dissociation between perception and action in the Ebbinghaus illusion. Curr Biol. 2001;11(3):177-81. doi: 10.1016/s0960-9822(01)00023-9. 
44. Notredame CE, Pins D, Deneve S, Jardri R. What visual illusions teach us about schizophrenia. Frontiers Integrat Neurosci. 2014;8 doi: 10.3389/fnint.2014.00063. 